How do sinking phytoplankton species manage to persist?

J. Huisman, M. Arrayás, U.M. Ebert, B.P. Sommeijer

Modelling, Analysis and Simulation (MAS)

MAS-R0105 July 31, 2001 
Report MAS-R0105

ISSN 1386-3703

CWI

P.O. Box 94079

1090 GB Amsterdam

The Netherlands

$\mathrm{CWI}$ is the National Research Institute for Mathematics and Computer Science. CWI is part of the Stichting Mathematisch Centrum (SMC), the Dutch foundation for promotion of mathematics and computer science and their applications.

SMC is sponsored by the Netherlands Organization for Scientific Research (NWO). CWI is a member of ERCIM, the European Research Consortium for Informatics and Mathematics.

Copyright (C) Stichting Mathematisch Centrum P.O. Box 94079, 1090 GB Amsterdam (NL) Kruislaan 413, 1098 SJ Amsterdam (NL) Telephone +3120 5929333 Telefax +31205924199 


\title{
How do Sinking Phytoplankton Species Manage to Persist?
}

\author{
Jef Huisman * \\ Aquatic Microbiology, Institute for Biodiversity and Ecosystem Dynamics \\ University of Amsterdam \\ Nieuwe Achtergracht 127, 1018 WS Amsterdam, The Netherlands \\ jef.huisman@chem.uva.nl \\ Manuel Arrayás ${ }^{\dagger} \quad$ Ute Ebert Ben Sommeijer \\ $C W I$ \\ P.O. Box 94079, 1090 GB Amsterdam, The Netherlands \\ manuel.arrayas@cwi.nl, ute.ebert@cwi.nl, bsom@cwi.nl
}

\begin{abstract}
Phytoplankton requires light for photosynthesis, but most phytoplankton species are heavier than water and sink. How can these sinking species persist? Here we show, by means of an advection-diffusion-reaction equation of light-limited phytoplankton, that the answer lies in the turbulent motion of water that re-disperses phytoplankton over the vertical water column. More specifically, we show that there is a turbulence window sustaining sinking phytoplankton species. If turbulent diffusion is too high, phytoplankton is mixed to great depths and the depth-averaged light conditions are too low to allow net positive population growth. Conversely, if turbulent diffusion is too low, sinking phytoplankton populations end up at the ocean floor and succumb in the dark. At intermediate levels of turbulent diffusion, however, the model predicts that phytoplankton populations can outgrow both mixing rates and sinking rates. In this way, the reproducing population as a whole can maintain a position in the well-lit zone near the top of the water column, even if all individuals within the population have a tendency to sink. This theory unites earlier classic results by Sverdrup and Riley as well as our own recent findings, and provides a novel conceptual framework for the understanding of phytoplankton dynamics under influence of mixing processes.
\end{abstract}

2000 Mathematics Subject Classification: Primary: 92B05, 92D25. Secondary: 65M20. 1998 ACM Computing Classification System: I.6.5, J.3, G.1.7 and G.1.8.

Keywords and Phrases: Phytoplankton blooms, Advection-diffusion-reaction equation, Critical depth, Critical diffusion, Export production, Light limitation, Numerical modelling.

Note: Work carried out under subthemes MAS1.3 - Applications from the Life Sciences

${ }^{*}$ Correspondence and requests for materials should be addressed to the first author.

†also: Lorentz Institute, University of Leiden, P.O. Box 9506, 2300 RA Leiden, The Netherlands 
and MAS1.4 - Pattern Formation and Low Temperature Plasmas.

J.H. and B.S. were supported by the Earth and Life Sciences Foundation (ALW), which is subsidized by the Netherlands Organization for Scientific Research (NWO). M.A. was supported by a TMR-grant from the European Union,

\section{Introduction}

Phytoplankton requires light for photosynthesis. Hence, in order to proliferate, phytoplankton populations should stay in the upper regions of the water column, the so-called euphotic zone. However, many if not most phytoplankton species have a higher specific weight than water. They sink $[14,27,22]$. Yet sinking phytoplankton species form a successful part of the phytoplankton community in lakes and oceans for millions of years. How do populations of sinking phototrophic organisms manage to persist? Which environmental factors allow survival of sinking phytoplankton?

Previous studies provided partial answers to these questions. In a classic paper, Riley et al. [23, page 90] derived a relation between sinking velocity and water-column turbulence that would just allow the persistence of a sinking phytoplankton population. Though Riley et al. focused on the interplay between sinking velocity and turbulent diffusion, their math neglected the light dependency of phytoplankton growth. Later, Shigesada and Okubo [25] reproduced the result of Riley et al. in a model in which they incorporated light-dependent growth rates but neglected light absorption by the water column. In another classic, Sverdrup [28] derived the existence of a "critical depth" of the mixed layer, beyond which phytoplankton growth would be impossible. Sverdrup considered light-dependent growth rates as well as light absorption by the water column, but assumed a uniform phytoplankton depth profile thereby neglecting implications of both turbulent diffusion and sinking. Sverdrup's critical-depth theory gained much impetus in modern oceanography and aquatic ecology (see e.g $[21,16,19,18,10]$ ). Recently, Huisman et al. $[11,12]$ derived the existence of a 'critical turbulence' that just allows phytoplankton bloom development. Huisman et al. focused on the interplay between turbulent diffusion and light-dependent growth rates, but neglected sinking of phytoplankton. How do these different concepts fit together? Is there any consistency or overlap between these theories? It feels as if we have different pieces of a complicated puzzle at hand, while the coherent picture is still lacking.

The issue is not without relevance. Sinking phytoplankton species play a key role in several biogeochemical cycles, as they withdraw nutrients from the upper part of the water column and deposit these nutrients at the bottom sediment. In particular, sinking phytoplankton species have a major impact on the global carbon cycle by their export of photosynthetic carbon from the surface into the deep ocean interior (see [7, 1, 4]). A better understanding of the population dynamics of sinking phytoplankton may thus contribute to a better understanding of the biogeochemical cycling of elements in aquatic ecosystems.

In this paper, we develop a population-dynamic theory of sinking phytoplankton. The theory is based on a reaction-advection-diffusion equation, that considers the balance between light-dependent growth rates, mortality rates, sinking rates, and turbulent diffusion rates. This reaction-advection-diffusion equation lies at the heart of a wide variety of 
detailed simulation models in oceanography and ecosystems research [15, 26, 24, 5, 18, 3]. Our results will show that the earlier theoretical concepts developed by Riley et al. [23], Sverdrup [28], and Huisman et al. [11] can be embraced into a single unifying theory.

\section{The model}

We consider a water column with a cross section of one unit area. Let $z$ denote the depth coordinate within the water column, where $z$ runs from 0 at the top to a maximum depth, $z_{m}$, at the bottom. Let $I(z, t)$ denote the light intensity at depth $z$ and time $t$, and let $\omega(z, t)$ denote the phytoplankton population density (cells per unit volume) at depth $z$ and time $t$.

Light gradient: Photons are absorbed by water, clay particles, phytoplankton, and many other light-absorbing substances. We assume that the light gradient $I$ follows LambertBeer's law, which states that light absorption at a depth $z$ is proportional to the local concentration of light absorbers at this depth:

$$
\frac{\partial I}{\partial z}(z, t)=-\left(k \omega(z, t)+K_{b g}\right) I(z, t),
$$

where $K_{b g}$ summarizes the total background attenuation due to all non-phytoplankton components, and $k$ is the specific light attenuation coefficient of the phytoplankton. Integrating this equation over depth gives the following light intensity at depth $z$ and time $t$ :

$$
I(z, t)=I_{i n} e^{-K_{b g} z} e^{-k \int_{0}^{z} \omega(\sigma, t) d \sigma},
$$

where $I_{i n}$ is the incident light intensity, and $\sigma$ is an integration variable. Note that this formulation includes light absorption by phytoplankton. Thus, the light gradient changes with a change in the phytoplankton population density distribution.

Local population dynamics: The changes in phytoplankton population density can be described by the partial differential equation

$$
\frac{\partial \omega}{\partial t}(z, t)=g(I(z, t)) \omega(z, t)-\frac{\partial J}{\partial z}(z, t) .
$$

Here $g(I(z, t))$ is the specific growth rate of phytoplankton as a function of the light intensity $I(z, t)$, and $J(z, t)$ is the vertical flux of phytoplankton at depth $z$ and time $t$. The minus sign indicates that an increase of the flux with depth implies a decrease of the local population density.

The specific growth rate in (3) depends on the balance between production and losses:

$$
g(I)=p(I)-\ell
$$

where $p(I)$ is the specific production rate as an increasing function of light intensity, with $p(0)=0$, and $\ell$ is the specific loss rate. In all our simulations, we used the following 
$p(I)$-function $[20,10]$ :

$$
p(I)=\frac{p_{\max } I}{H+I}
$$

where $p_{\max }$ is the maximal specific production rate and $H$ is a half-saturation constant. We emphasize, however, that the results presented in this paper rely on the qualitative behavior of the $p(I)$-relation (i.e., $p(0)=0$ and $\frac{d}{d I} p(I)>0$ ), rather than on its specific form.

The flux of phytoplankton in (3) depends on the sinking rate of phytoplankton and on transport of phytoplankton by turbulent diffusion:

$$
J(z, t)=v \omega(z, t)-D \frac{\partial \omega}{\partial z}(z, t),
$$

where $v$ is the vertical velocity of the phytoplankton, and $D$ is the turbulent diffusion coefficient. In this paper, both $v$ and $D$ will be assumed constant. The positive sign of the first term on the right-hand side of (6) implies that $v$ is positive for sinking phytoplankton. The minus sign in the second term on the right-hand side indicates that turbulent diffusion is in the direction opposite to the concentration gradient.

Substituting (2), (4) and (6) into (3) yields our key equation

$$
\frac{\partial \omega}{\partial t}=p\left(I_{i n} e^{-K_{b g} z} e^{-k \int_{0}^{z} \omega(\sigma, t) d \sigma}\right) \omega-\ell \omega-v \frac{\partial \omega}{\partial z}+D \frac{\partial^{2} \omega}{\partial z^{2}} .
$$

This is an integro-partial differential equation. The first term on the right-hand side indicates that the specific production rate at a certain depth depends on the light intensity at this depth, which in turn depends (via Lambert-Beer's law) on all population densities above this depth.

Boundary conditions: We assume that the boundaries of the system are closed:

$$
J(0, t)=v \omega(0, t)-D \frac{\partial \omega}{\partial z}(0, t)=0,
$$

and

$$
J\left(z_{m}, t\right)=v \omega\left(z_{m}, t\right)-D \frac{\partial \omega}{\partial z}\left(z_{m}, t\right)=0 .
$$

That is, there is no influx or efflux of phytoplankton, neither at the top nor at the bottom of the water column.

Total population dynamics: It is useful to keep track not only of the local population densities but also of the total phytoplankton population in the entire water column. This can be expressed as the total population size per unit surface area, $W$, defined by

$$
W(t)=\int_{0}^{z_{m}} \omega(z, t) d z .
$$


Hence, using (3) and the boundary conditions, the population size per unit surface area changes with time according to

$$
\frac{d W}{d t}=\int_{0}^{z_{m}} g(I(z, t)) \omega(z, t) d z
$$

where the flux terms canceled because the boundaries are closed.

Numerical simulations: The model predictions are analyzed using a combination of analytical and numerical techniques. Numerical simulation of integro-partial differential equations is quite challenging. Sections 6 and 7 give a detailed description of the simulation techniques that we have employed.

\section{Inert particles and the barometric formula}

Before analyzing the model predictions for sinking phytoplankton, let us first investigate the fate of sinking inert particles. Inert particles are particles that neither grow nor decay. That is, $p(I)=0$ and $\ell=0$. Because the boundaries are closed, particles cannot enter or leave the water column. Hence, transport by sinking and turbulent mixing does not affect the total number of inert particles within the water column but does affect their vertical distribution. Using (7), the population dynamics for inert particles reads

$$
\frac{\partial \omega}{\partial t}(z, t)=-v \frac{\partial \omega}{\partial z}(z, t)+D \frac{\partial^{2} \omega}{\partial z^{2}}(z, t) .
$$

The slope of the stationary population density distribution of the inert particles is obtained by solving (11) for steady state (i.e., solving for $\partial \omega / \partial t=0$ ), and subsequent integration over depth. We notice from the boundary conditions in (8a) and (8b) that the constant of integration equals zero. Thus, the slope of the stationary depth profile is given by

$$
\frac{d \omega^{*}}{d z}(z)=\frac{v}{D} \omega^{*}(z)
$$

where the superscript $*$ indicates that we consider a stationary distribution. Integrating over depth once more, it follows that the stationary population density distribution of inert particles is given by

$$
\omega^{*}(z)=\omega^{*}(0) \exp \left(\frac{v}{D} z\right)
$$

Recalling that $v>0$ for sinking particles, we have arrived at a surprisingly simple result: the stationary population density distribution of sinking inert particles is an exponentially increasing function of depth. The steepness of the depth profile depends on the ratio of sinking velocity versus turbulent diffusion. If turbulent diffusion is high and sinking velocity is low, the inert particles will be almost uniformly distributed over the water column. Conversely, if turbulent diffusion is low and sinking velocity is high, the inert particles will concentrate at the bottom of the water column.

A similar derivation applies to the density distribution of gas molecules in the atmosphere. Therefore, Equation (13) is widely known in physics as the Barometric Formula (see e.g. [17]). 


\section{Phytoplankton depth profiles}

A key difference between inert particles and phytoplankton is that the latter reproduce in the light and suffer net losses in the dark. Hence, the question arises: What will be the stationary depth profiles for sinking phytoplankton?

One solution for the stationary depth profile is, of course, that there is no phytoplankton at any depth at all. More precisely, $\omega^{*}(z)=0$ for all $z$ is, indeed, a trivial stationary solution of (7). Below we consider the non-trivial case in which a phytoplankton population does develop.

According to the boundary condition in $(8 \mathrm{a})$, if $\omega^{*}(0)>0$, the stationary population density is an increasing function of depth in top of the water column:

$$
\frac{d \omega^{*}}{d z}(0)=\frac{v}{D} \omega^{*}(0)
$$

Similarly, if $\omega^{*}\left(z_{m}\right)>0$, population density is an increasing function of depth at the bottom of the water column as well:

$$
\frac{d \omega^{*}}{d z}\left(z_{m}\right)=\frac{v}{D} \omega^{*}\left(z_{m}\right)
$$

because of the boundary condition in (8b). Thus, at the top and bottom of the water column, the stationary depth profile of phytoplankton resembles the stationary depth profile of inert particles (compare (14a) and (14b) with (12)).

The slope of the stationary depth profile at intermediate depths can be derived by solving (7) for the stationary distribution, and subsequent integration over depth. The constant of integration is determined by (14a), and the result is

$$
\frac{d \omega^{*}}{d z}(z)=-\frac{1}{D} \int_{0}^{z} g(I(\sigma)) \omega^{*}(\sigma) d \sigma+\frac{v}{D} \omega^{*}(z)
$$

The first term on the right-hand side of (15) equals zero at $z=0$ and at $z=z_{m}$, as is obvious at $z=0$ and follows from (14b) at $z=z_{m}$. Using the monotonicity of the $g(I(\sigma))$-function, it follows that the integral $\int_{0}^{z} g(I(\sigma)) \omega^{*}(\sigma) d \sigma$ is positive for $0<z<z_{m}$. The second term on the right-hand side of $(15)$ is positive. Thus, whether the slope of the stationary depth profile is positive or negative at intermediate depths depends on the magnitudes of the two opposing terms in (15). That is, the slope of the depth profile depends on the magnitude of the ratio between depth-integrated growth rate and turbulent diffusion versus the magnitude of the ratio between sinking flux and turbulent diffusion. Accordingly, the population will be uniformly distributed over depth if turbulent diffusion overrides the difference between depth-integrated growth rates and sinking fluxes. Population density will increase with depth if sinking fluxes override depth-integrated growth rates. Conversely, population density will decrease with depth if depth-integrated growth rates override sinking fluxes.

Figures 1-3 show a variety of stationary depth profiles illustrating these derivations. In Figures 1A-1D, the turbulent diffusion coefficient is gradually increased. This shows that the stationary depth profile can have a local population density maximum below 
Table 1: Parameter values used in the simulations

\begin{tabular}{llll}
\hline Symbol & Meaning & Value & Units \\
\hline Variables & light intensity & & \\
$I$ & vertical flux of phytoplankton & & $\mu \mathrm{mol} \mathrm{photons} \cdot \mathrm{m}^{-2} \cdot \mathrm{s}^{-1}$ \\
$J$ & population density $\cdot \mathrm{m}^{-2} \cdot \mathrm{h}^{-1}$ & $\mathrm{cells} \cdot \mathrm{m}^{-3}$ \\
$\omega$ & population size & & $\mathrm{cells} \cdot \mathrm{m}^{-2}$ \\
$W$ & & & \\
& & & \\
Parameters & & 1 & $\mathrm{~cm}^{2} \cdot \mathrm{s}^{-1}$ \\
$D$ & turbulent diffusion & 350 & $\mu \mathrm{mol} \mathrm{photons} \cdot \mathrm{m}^{-2} \cdot \mathrm{s}^{-1}$ \\
$I_{\text {in }}$ & incident light intensity & 0.2 & $\mathrm{~m}^{-1}$ \\
$K_{b g}$ & background turbidity & & \\
$k$ & specific light attenuation & & \\
& of phytoplankton & $15 \cdot 10^{-12}$ & $\mathrm{~m}^{2} \cdot \mathrm{cell}^{-1}$ \\
$\ell$ & specific loss rate & 0.01 & $h^{-1}$ \\
$p(I)$ & specific production rate & $(*)$ & $h^{-1}$ \\
$v$ & vertical velocity & 0.04 & $\mathrm{~m} \cdot \mathrm{h}^{-1}$ \\
$z_{m}$ & water column depth & 20 & $\mathrm{~m}$ \\
\hline
\end{tabular}

$(*)$ In all our simulations, the specific production rate was described by Equation (5) with $p_{\text {max }}=0.04 h^{-1}$ and $H=30 \mu \mathrm{mol}$ photons $\cdot m^{-2} \cdot s^{-1}$.

the surface as well as accumulation of phytoplankton near the bottom of the water column. This occurs if depth-integrated growth rates exceed sinking and turbulent mixing (Fig. 1A, B). The depth profiles become less pronounced with increasing turbulent diffusion (Fig. 1C, D). In Figures 2A-2D, the sinking velocity is gradually increased. This shows that the stationary phytoplankton population maintains a position near the surface when sinking velocity is low (Fig. 2A, B), and shifts downwards with increasing sinking velocity (Fig. 2C,D). In Figures 3A-3D, the depth of the water column is gradually increased. This illustrates clearly that, in deep waters, sinking phytoplankton populations can maintain a position in the upper part of the water column independent of the water column depth (Fig. 3C,D).

\section{Conditions for bloom development}

\subsection{General findings}

The stationary depth profiles in Figures 1-3 are all positive (i.e., $W^{*}>0$ ). In this case, we say that there is 'bloom development'. Alternatively, the stationary population density distribution might be zero at all depths (i.e., the trivial solution $W^{*}=0$ ). In this case, we say that there is 'no bloom'. What are the conditions favorable for bloom development of sinking phytoplankton?

Figure 4A shows regions of bloom development and regions of no blooms, plotted for a wide range of different water column depths and turbulent diffusivities. Note the log scales of the axes: the graphs span the entire spectrum from shallow, quiescent lakes to 


\section{Population density $\left(10^{4}\right.$ cells $\left.\mathrm{ml}^{-1}\right)$}
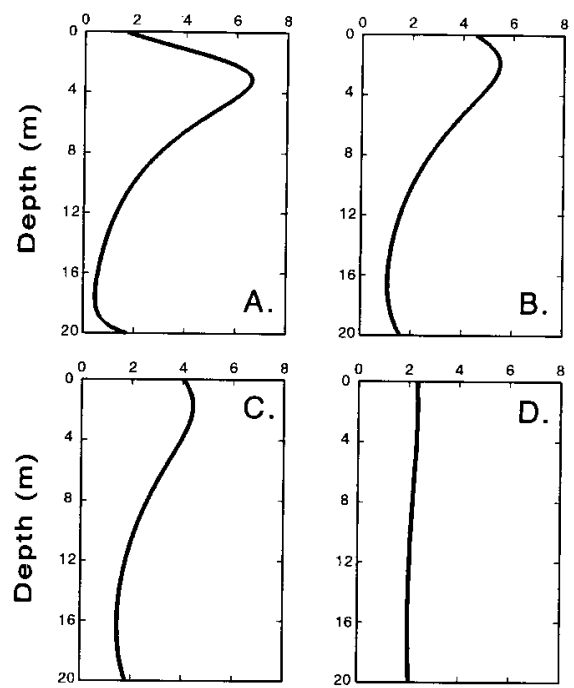

Figure 1: Stationary depth profiles of sinking phytoplankton, for four different turbulence levels. Parameter values as in Table 1, except for the turbulent diffusion coefficients: (A) $D=0.1 \mathrm{~cm}^{2} \cdot \mathrm{s}^{-1}$, (B) $D=0.5 \mathrm{~cm}^{2} \cdot \mathrm{s}^{-1}$, (C) $D=1 \mathrm{~cm}^{2} \cdot \mathrm{s}^{-1}$, (D) $D=10 \mathrm{~cm}^{2} \cdot \mathrm{s}^{-1}$.

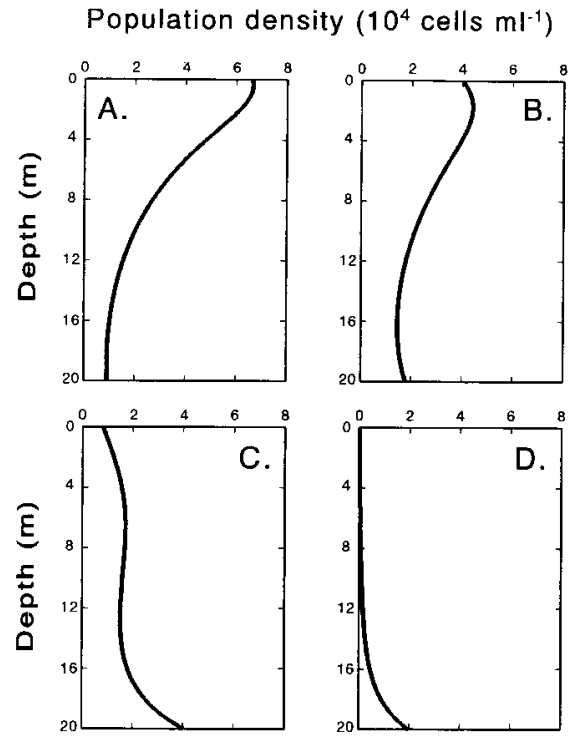

Figure 2: Stationary depth profiles of sinking phytoplankton, for four different sinking velocities. Parameter values as in Table 1, except for the sinking velocities: (A) $v=0.01 \mathrm{~m} \cdot \mathrm{h}^{-1}$, (B) $v=0.04 m \cdot h^{-1}$, (C) $v=0.10 m \cdot h^{-1}$. (D) $v=0.15 m \cdot h^{-1}$. 


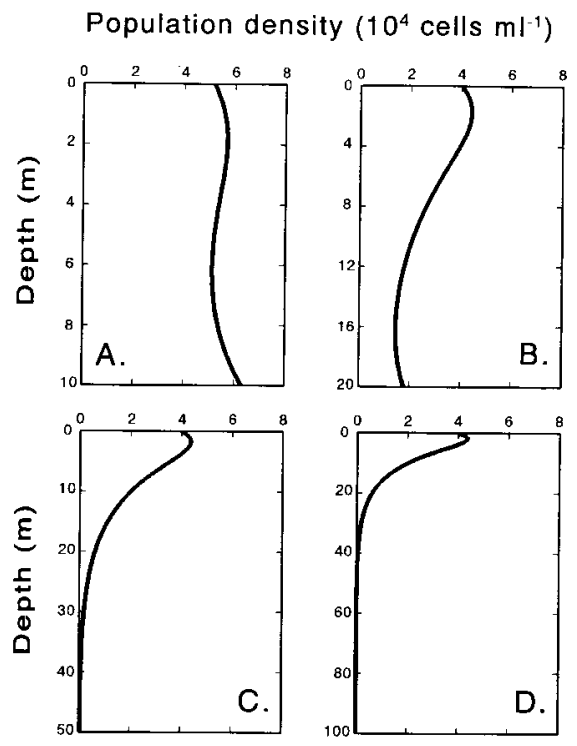

Figure 3: Stationary depth profiles of sinking phytoplankton, for four different water-column depths. Parameter values as in Table 1, except for the water-column depths: (A) $z_{m}=10 m$, (B) $z_{m}=20 \mathrm{~m},(\mathrm{C}) z_{m}=50 \mathrm{~m},(\mathrm{D}) z_{m}=100 \mathrm{~m}$.

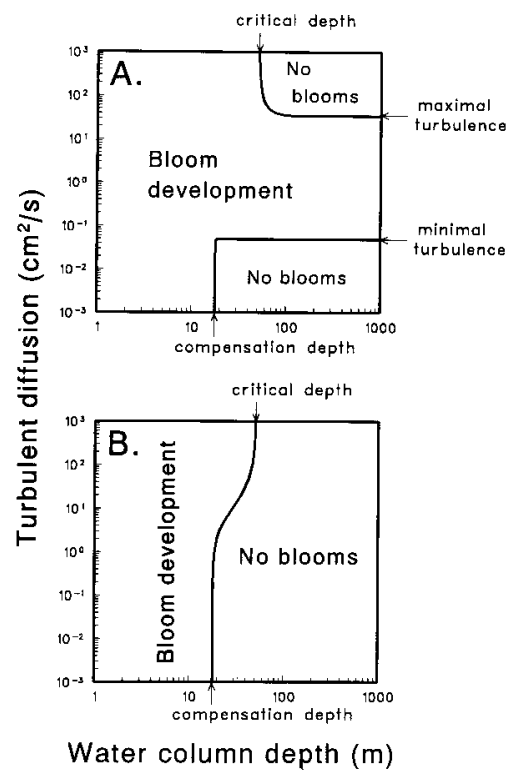

Figure 4: Combinations of water-column depth and turbulent diffusion coefficient that allow a phytoplankton bloom, and combinations that prevent a phytoplankton bloom. (A) A phytoplankton species with a moderate sinking velocity of $v=0.04 m \cdot h^{-1}$, (B) A phytoplankton species with a high sinking velocity of $v=0.40 \mathrm{~m} \cdot \mathrm{h}^{-1}$. The graphs are each based on a grid of $31 \times 61=1,891$ simulations. Parameter values as in Table 1. 


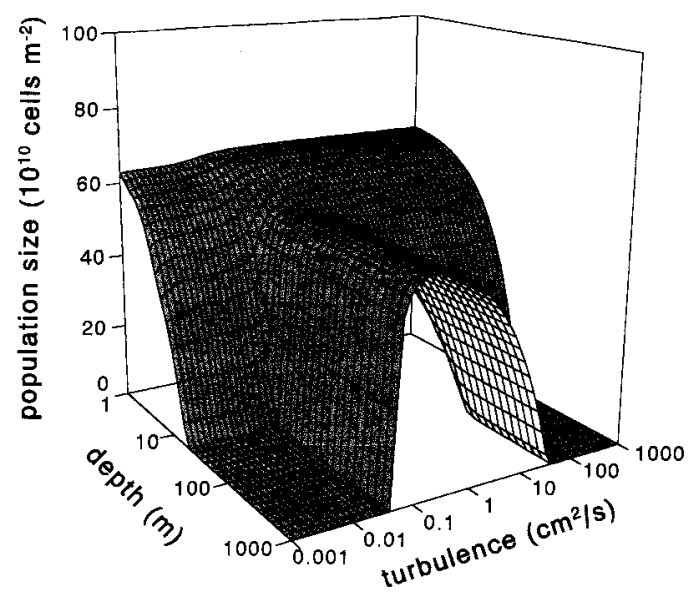

Figure 5: Total population size per unit surface area, $W$, as a function of water-column depth and turbulent diffusion coefficient. The graph is based on a grid of $31 \times 60=1,891$ simulations. Parameter values as in Table 1.

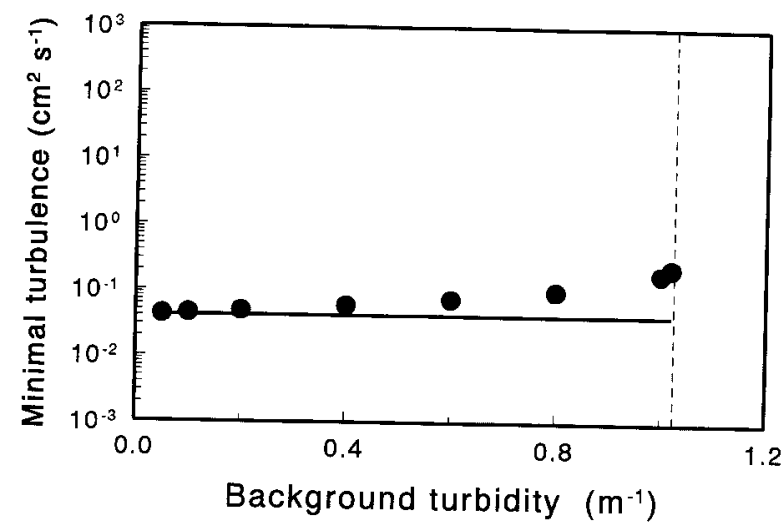

Figure 6: Minimal turbulence as a function of background turbidity, predicted by the full model (dots) and predicted by Equation (19) (solid line). Phytoplankton blooms cannot be sustained if background turbidity exceeds the dashed line. Parameter values as in Table 1. 
deep, turbulent oceans. The left part of Fig. 4A considers shallow waters. In shallow waters there is sufficient light for bloom development, irrespective of the phytoplankton distribution over depth. Hence, blooms can develop. The upper right corner of Fig. 4A considers deep waters with a high turbulent mixing rate. Here, phytoplankton is mixed uniformly over great depths, and the average light conditions over the entire water column are insufficient for a net positive growth rate. Hence, blooms do not develop. The lower right corner of Fig. $4 \mathrm{~A}$ considers deep waters with a low turbulent mixing rate. Here, sinking rates exceed growth rates and mixing rates, and phytoplankton sinks downwards to great depths. Consequently, blooms do not develop either. Most surprisingly, however, blooms of sinking species can develop in deep systems with intermediate mixing rates (middle right of Fig. 4A). In this parameter region, growth rates exceed mixing rates so that uniform mixing over the entire depth of the water column is prevented. Moreover, turbulent mixing rates exceed sinking rates so that large downward fluxes of phytoplankton are avoided. As a consequence, sinking species can maintain a population in the euphotic zone at intermediate mixing rates.

Interestingly, the 'no bloom' areas in Fig. $4 \mathrm{~A}$ are bound by nearly horizontal and vertical lines. This implies that the effects of water column depth and turbulent diffusion on phytoplankton bloom development can be considered independently of one another. Hence, we can recognize a 'critical depth', a 'compensation depth', a 'maximal turbulence', and a 'minimal turbulence' (Fig. 4A). We call the region between the maximal and minimal turbulence, the 'turbulence window' for sinking phytoplankton.

Figure 5 plots the total population size per unit surface area, $W$, as a function of water column depth, $z_{m}$, and turbulent diffusion coefficient, $D$. The two 'no bloom' regions at low and high turbulence are clearly visible (compare Fig. 5 with Fig. 4A). If the water column is shallow $\left(z_{m}<10\right.$ meters), population size per unit surface area is a decreasing function of water column depth, whereas it is independent of turbulence. Conversely, if the water column is deep $\left(z_{m}>50\right.$ meters), population size per unit surface area is a unimodal function of turbulence, whereas it is independent of water column depth. The two patterns are essentially perpendicular to each other. This provides another illustration of the phase transition documented in this paper: Water column depth determines the population size of phytoplankton blooms in shallow systems, whereas turbulence determines the population size of phytoplankton blooms in deep systems.

\subsection{Explicit expressions}

It would be convenient, for both practical applications and a better general understanding, to have a fast method available to calculate the four critical parameters of Fig. 4A. We developed two procedures. Firstly, we derived a fast and accurate numerical algorithm, described in Section 7. Secondly, we derived analytical expressions for the four critical parameters, which are discussed below.

\subsubsection{Critical depth}

The critical depth in Figure 4 is equivalent to Sverdrup's [28] concept of a critical depth. Intuitively, the idea is that, in turbulent waters, phytoplankton is uniformly mixed (as in 
Fig. 1D) and therefore have high production rates in the upper part of the water column but suffer losses throughout the water column. Hence, depth-integrated production rates may be less than depth-integrated loss rates if the water column becomes too deep. That is, bloom development in turbulent waters is impossible if water column depth exceeds a critical depth.

The critical depth, $z_{c r}$, can be written as [10]

$$
z_{c r}=\frac{\ln \left(I_{\text {in }}\right)-\ln \left(I_{\text {out }}^{*}\right)}{K_{b g}}
$$

where $I_{\text {out }}^{*}$ is the critical light intensity, which is defined by the implicit equation [13]

$$
\int_{I_{\text {out }}^{*}}^{I_{\text {in }}} \frac{g(I)}{k I} d I=0 .
$$

The critical light intensity corresponds to the light intensity, measured at the bottom of a water column, for which a total phytoplankton population uniformly distributed throughout the water column would remain stationary $[13,10]$.

\subsubsection{Compensation depth}

The compensation depth, $z_{C}$, in Figure 4 is the depth at which the compensation light intensity would be reached in the absence of phytoplankton. That is,

$$
z_{C}=\frac{\ln \left(I_{i n}\right)-\ln \left(I_{C}\right)}{K_{b g}}
$$

where the compensation light intensity, $I_{C}$, corresponds to the light intensity at which specific production rate equals specific loss rate. That is, the compensation light intensity is defined by $g\left(I_{C}\right)=0$. Intuitively, the idea is that, in systems with a low turbulence, the total phytoplankton population sinks to the bottom of the water column (as in Fig. 2D) and growth conditions thus depend only on the light conditions at the bottom. Equation (18) states that a phytoplankton population located at the bottom of the water column cannot develop a bloom if light conditions at the bottom are insufficient for production rates to exceed loss rates. Thus, bloom development in quiet waters is impossible if water column depth exceeds the compensation depth.

We note that the critical depth is always deeper than the compensation depth (i.e., the critical light intensity is always lower than the compensation light intensity [13].

\subsubsection{Maximal turbulence}

The idea that underlies the maximal turbulence is that if turbulent diffusion is less than this maximal threshold value, phytoplankton populations may outgrow the turbulent mixing rate and may thus maintain a bloom in the upper part of the water column. Generally speaking, there is no simple analytical equation for the maximal turbulence. In the special case that the specific production rate is of the form $p(I)=a I^{\alpha}$, with $0<\alpha \leq 1$, we have been able to derive an implicit equation for the maximal turbulence based on 
so-called Bessel functions. Using asymptotic expansion techniques, these functions have been approximated to obtain a more tangible form [6]. We remark that the linear case $p(I)=a I$, where $\alpha=1$, is included in the above analysis. For nonlinear $p(I)$-functions with saturating properties, such as Equation (5), we recommend calculating the maximal turbulence by the numerical algorithm outlined in Section 6 .

\subsubsection{Minimal turbulence}

The idea of a minimal turbulence is that if turbulence becomes too low, there is no force that prevents sinking of the entire phytoplankton population. Hence, if turbulence is too low and the water column is deep, the entire phytoplankton population will be lost from the euphotic zone and vanishes in the dark. As for the maximal turbulence, a simple exact equation for the minimal turbulence does not exist. However, we can approximate the minimal turbulence by the ratio of the square of sinking velocity over four times the net specific growth rate near the water surface:

$$
D_{\min } \approx \frac{v^{2}}{4 g\left(I_{i n}\right)} .
$$

To derive this approximation, we first simplify the model considerably. Suppose that the water column can be separated into two layers. Throughout the top layer, there is ample light available for phytoplankton growth, and the specific growth rate equals $g\left(I_{i n}\right)>0$ in the entire top layer. In contrast, in the bottom layer there is no light available at all, and the growth rate equals $g(0)<0$ in the entire bottom layer. Furthermore, we assume that transport of phytoplankton within the bottom layer is governed by sinking only whereas turbulence is negligible. Therefore, once phytoplankton sinks from the bright top layer into the dark bottom layer, it cannot return. Continuity of the phytoplankton flux at the interface between the top layer and the bottom layer requires

$$
\lim _{z \downarrow z_{T}} v \omega(z, t)-D \frac{\partial \omega}{\partial z}(z, t)=\lim _{z \uparrow z_{T}} v \omega(z, t),
$$

where $z_{T}$ is the depth at which the interface is located. Incorporating these simplifying assumptions, we arrive at the following stationary equation for phytoplankton in the top layer:

$$
g\left(I_{i n}\right) \omega^{*}-v \frac{d \omega^{*}}{d z}+D \frac{d^{2} \omega^{*}}{d z^{2}}=0,
$$

with the boundary conditions, from (8a) and (20),

$$
\begin{gathered}
v \omega^{*}(z, t)-D \frac{d \omega^{*}}{d z}(z, t)=0, \quad \text { at } z=0, \\
\frac{d \omega^{*}}{d z}(z, t)=0, \quad \text { at } z=z_{T} .
\end{gathered}
$$


Equation (21) is a linear and homogeneous second-order differential equation with constant coefficients. Thus, its characteristic equation has two roots

$$
\begin{aligned}
& r_{1}=\frac{v}{2 D}+\frac{\sqrt{v^{2}-4 g\left(I_{i n}\right) D}}{2 D}, \\
& r_{2}=\frac{v}{2 D}-\frac{\sqrt{v^{2}-4 g\left(I_{i n}\right) D}}{2 D} .
\end{aligned}
$$

Suppose that $v^{2}>4 g\left(I_{i n}\right) D$. Then the distinct roots $r_{1}$ and $r_{2}$ are positive and real. Hence, the stationary population density profile reads

$$
\omega^{*}(z)=c_{1} e^{r_{1} z}+c_{2} e^{r_{2} z},
$$

where $c_{1}$ and $c_{2}$ are constants of integration. Combining (24) with the boundary condition (22a) implies

$$
v\left(c_{1}+c_{2}\right)-D\left(r_{1} c_{1}+r_{2} c_{2}\right)=0 .
$$

Combining (24) with the boundary condition (22b) implies

$$
r_{1} c_{1} e^{r_{1} z_{T}}+r_{2} c_{2} e^{r_{2} z_{T}}=0
$$

Equations (25) and (26) are incompatible, unless $c_{1}=c_{2}=0$. But if $c_{1}=c_{2}=0$, then the stationary population density profile is zero throughout the water column. In other words, under the supposition $v^{2}>4 g\left(I_{i n}\right) D$, a bloom cannot develop. Thus, a necessary condition for bloom development is $v^{2}<4 g\left(I_{i n}\right) D$. This yields (19).

Equation (19) is equivalent to the classic equation derived by Riley et al. [23, page 90] (see also [25]), though these authors used a different model formulation. We emphasize that (19) is only an approximation. For instance, the above derivation neglects the background turbidity of the water column. Numerical simulation of the full model, however, indicates that (19) is an accurate approximation of the minimal turbulence in waters with a low background turbidity. The minimal turbulence is somewhat higher than predicted by (19) in waters with a high background turbidity (see Figure 6 ). Thus, to calculate the minimal turbulence, we may recommend (19) for waters with a low background turbidity and we may recommend the fast algorithm outlined in Section 7 for waters with a high background turbidity.

\subsection{Effects of sinking velocity}

We note, from Equations (16-18), that the critical depth and compensation depth are both independent of the sinking velocity of phytoplankton. In contrast, according to (19), the minimal turbulence increases with the square of phytoplankton sinking velocity. Moreover, numerical simulations indicate that the maximal turbulence decreases with sinking velocity. Therefore, if the sinking velocity of phytoplankton is too high, the minimal turbulence and maximal turbulence merge and disappear. Thus, while phytoplankton species with a moderate sinking speed can persist in deep waters (Fig. 4A), phytoplankton species with a high sinking speed cannot persist in deep waters (Fig. 4B). 


\section{Numerical simulation of the integro-PDE}

To obtain a fully discrete solution in space and time we will follow the so-called Method of Lines approach. That is, first the spatial differential operators (as well as the integral term) will be replaced by discrete approximations and subsequently the resulting system of ordinary differential equations (ODEs) - which is still continuous in time - will be integrated numerically.

\subsection{Spatial discretization}

First, we define a spatial grid on the interval $0 \leq z \leq z_{m}$. In case a priori knowledge about the solution is available, the grid can be chosen with relatively many points in regions where a high spatial activity is expected. In fact, the software that we developed indeed offers this facility. However, to avoid unnecessary complications in the description, the numerical procedure will be explained on the basis of an equidistant grid.

Hence, let us define:

$$
s_{0}=0, \quad s_{i}=\left(i-\frac{1}{2}\right) \Delta z, \quad i=1, \ldots, N, \quad s_{N+1}=z_{m},
$$

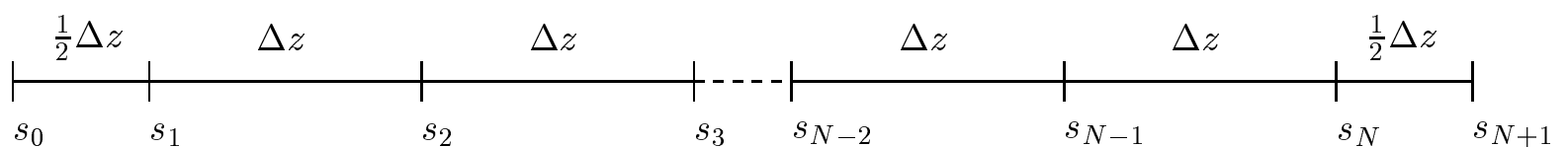

where $\Delta z:=z_{m} / N$. Furthermore, by $w_{i}(t), i=1, \ldots, N$, we denote an approximation to $\omega\left(s_{i}, t\right)$. To discretize in the point $s_{i}$ the derivative of the flux, i.e., the term $\partial J(z, t) / \partial z$ in Equation (3), we assume an (imaginary) box around the point $s_{i}$, at the boundaries of which we approximate the fluxes, using the numerical values $w_{i}$ at the grid points:

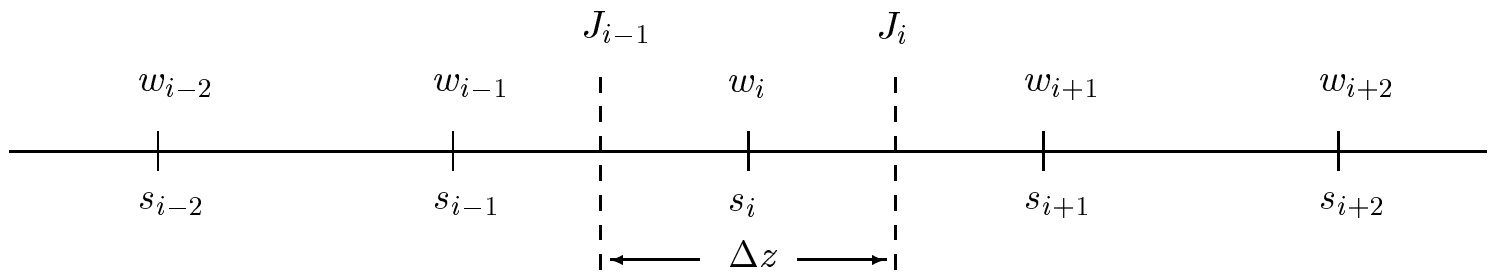

In this way we obtain conservation of the quantity $J$ since the numerical approximation that we use for a particular $J_{i} \approx J\left(s_{i}+\frac{1}{2} \Delta z, t\right)$ serves as outflow for one particular box and at the same time as inflow for the adjacent box. Now, $\partial J(z, t) / \partial z$ in the point $z=s_{i}$ is approximated by $\left(J_{i}-J_{i-1}\right) / \Delta z$. Next, we need an approximation for $J_{i}$. Here we use the approach that is nowadays standard in the field of Computational Fluid Dynamics for the numerical solution of advection-diffusion equations. That is, the diffusion term is discretized symmetrically, whereas for the advection term a so-called upwind (or, upstream) discretization is used (see e.g., [9]). To be more precise, in case of sinking, where the flow 
is from left to right in the above figure, we use (cf. Equation (6))

$$
\begin{aligned}
J_{i} & =v \omega\left(s_{i}+\frac{1}{2} \Delta z, t\right)-D \frac{\partial \omega}{\partial z}\left(s_{i}+\frac{1}{2} \Delta z, t\right) \\
& \approx v \frac{1}{6}\left[-w_{i-1}+5 w_{i}+2 w_{i+1}\right]-D \frac{w_{i+1}-w_{i}}{\Delta z} .
\end{aligned}
$$

This expression explains the term 'upwind': we see that more information from the left (i.e., the upstream region in case of sinking) has been used. Analogously, in case of buoyancy, we again use an upwind discretization, now using more information from the right, resulting in the approximation

$$
J_{i} \approx v \frac{1}{6}\left[2 w_{i}+5 w_{i+1}-w_{i+2}\right]-D \frac{w_{i+1}-w_{i}}{\Delta z} .
$$

We remark that a symmetric discretization of the advection term easily leads to so-called 'wiggles' in the numerical solution. By this we mean that, in the neighbourhood of a drastic change in the solution, undershoot and overshoot values will be developed. Especially in case of undershoot (i.e., too low values) this may lead to negative solution components. A negative population density is of course not realistic. The use of upwind techniques drastically reduces this unwanted property.

The fluxes $J_{0}$ and $J_{N}$ vanish according to the boundary conditions and for $J_{1}$ we use a symmetric formula since we lack sufficient upstream information (in case of sinking).

Summarizing, for sinking phytoplankton, we arrive at the following set of ODEs

$$
\frac{d w_{i}(t)}{d t}=g_{i} w_{i}-\frac{J_{i}-J_{i-1}}{\Delta z}, \quad i=1, \ldots, N
$$

where

$$
\begin{aligned}
J_{0} & =0 \\
J_{1} & =v \frac{w_{2}+w_{1}}{2}-D \frac{w_{2}-w_{1}}{\Delta z}, \\
J_{i} & =v \frac{1}{6}\left[-w_{i-1}+5 w_{i}+2 w_{i+1}\right]-D \frac{w_{i+1}-w_{i}}{\Delta z}, \quad i=2, \ldots, N-1, \\
J_{N} & =0 .
\end{aligned}
$$

The term $g_{i}$ in (31) is defined as $g_{i}:=p\left(I_{i}\right)-l$, where $I_{i}$ denotes the light intensity at $z=s_{i}$. Replacing the integral term by the repeated trapezoidal rule, the light intensity is approximated by

$$
I_{i}=I_{\text {in }} e^{-K_{b g} s_{i}} e^{-k\left[\frac{1}{2} w_{0}+w_{1}+w_{2}+\ldots+w_{i-1}+\frac{1}{2} w_{i}\right] \Delta z},
$$

with the solution at the surface, $w_{0}$, extrapolated from inside: $w_{0}:=\left(3 w_{1}-w_{2}\right) / 2$.

\subsection{Time integration}

After spatial discretization we arrive at a large system of ODEs, written in the form

$$
\frac{d \mathbf{w}(t)}{d t}=\mathbf{F}(\mathbf{w}(t)), \quad t \geq 0
$$


where the vector $\mathbf{w}(t)$ contains the components $w_{i}(t)$. Our first observation is that this system is a stiff ODE. This means that the Jacobian matrix $\partial \mathbf{F} / \partial \mathbf{w}$ has widely spread eigenvalues (for a discussion on stiffness we refer to [8]). Stiffness has a direct consequence on the choice of the time integration technique. An explicit method, which is simple and cheap per step, would be forced by the stiff system to take small time steps in order to avoid instabilities. This time step restriction is in our application so severe that it is unfeasible to use an explicit integration method. Therefore, we selected an implicit method. Although such methods, in general, have good stability properties, we are now faced with the task to solve, in each time step, a system of implicit relations to obtain the solution at the next point in time. For the family of implicit methods that we have used, this results in solving the equation

$$
\mathbf{R}\left(\mathbf{W}_{n+1}\right):=\mathbf{W}_{n+1}-b_{0} \Delta t \mathbf{F}\left(\mathbf{W}_{n+1}\right)-\sum_{i=1}^{m} b_{i} \mathbf{W}_{n+1-i}=0
$$

Here, $\mathbf{W}_{n+1}$ is an approximation to $\mathbf{w}(t)$ at $t=t_{n+1}:=(n+1) \Delta t, \Delta t$ being the time step, and the coefficients $b_{i}$ are defined by the method in use. $\mathbf{W}_{n}, \mathbf{W}_{n-1}, \ldots, \mathbf{W}_{n+1-m}$ are approximate solutions at previous points in time and serve to give the method the required accuracy.

As usual, (34) is iteratively solved by means of Newton's process. That is, a series of linear systems of the form

$$
\left[I-b_{0} \Delta t \partial \mathbf{F} / \partial \mathbf{w}\right]\left[\mathbf{W}_{n+1}^{j}-\mathbf{W}_{n+1}^{j-1}\right]=-\mathbf{R}\left(\mathbf{W}_{n+1}^{j-1}\right), \quad j=0,1, \ldots
$$

have to be solved, where $I$ denoted the identity matrix. The iterates $\mathbf{W}_{n+1}^{j}$ hopefully converge to the solution $\mathbf{W}_{n+1}$.

The Jacobian matrix $\partial \mathbf{F} / \partial \mathbf{w}$ is composed of a 4-diagonal band (originating from the discretization of the advection-diffusion terms) plus a lower triangular part (due to the integral term). This makes the solution of the linear systems in (35) in the Newton process very time-consuming. To improve the numerical efficiency, we neglected the lower triangular part in the Jacobian matrix. As a result, the total number of Newton iterations (summed over all steps) increased by $30-50 \%$, but this is amply compensated by the strongly reduced costs to solve the linear systems (which now have a simple band structure).

Brown et al. [2] implemented the above numerical time integration technique in their code VODE, which we have used to produce the results described in the present paper. This code belongs to the family of most widely used stiff ODE solvers and is freely available from http://www.netlib.org/ode/ (both in Fortran and C). VODE is very robust in the sense that it includes all kind of strategies, necessary for automatic integration and incorporates experience of many users over a long period.

\section{A fast algorithm}

To estimate the critical depths and critical turbulences, one could run the integro-partial differential equation (integro-PDE) until steady state for a couple of thousand times in a 
fine grid of $z_{m}$-values and $D$-values, using the numerical simulation techniques outlined in the preceding section. However, this is a very time-consuming procedure and requires quite some programming skills. Therefore, as an alternative, this section develops a simple algorithm to calculate the critical depths and critical turbulences. The algorithm is fast, accurate, and very easy to apply. The trick is that, on the boundary line between the 'bloom' and 'no bloom' region (see Figure 4), the integro-PDE can be reduced to a secondorder ordinary differential equation (ODE).

\subsection{Development of the method}

We consider the transition from the 'bloom' to the 'no-bloom' area. At this transition, the population density of phytoplankton is negligibly small. More precisely, at this transition and also in the 'no-bloom' area itself, we have $\int_{0}^{z} k \omega^{*}(\sigma) d \sigma \ll K_{b g} z$. Hence, the light intensity $I$ can be approximated by $I_{i n} \exp \left(-K_{b g} z\right)$. This implies that the equation defining the stationary population density distribution reduces to a second-order ODE without an integro-term:

$$
g\left(I_{i n} e^{-K_{b g} z}\right) \omega^{*}(z)-v \frac{d \omega^{*}}{d z}(z)+D \frac{d^{2} \omega^{*}}{d z^{2}}(z)=0,
$$

which can be easily solved numerically. Equation (36) can be written as a system of two coupled first-order ODEs:

$$
\begin{aligned}
& \frac{d \omega^{*}}{d z}=\bar{\omega}^{*}, \\
& \frac{d \bar{\omega}^{*}}{d z}=-\frac{1}{D} g\left(I_{i n} e^{-K_{b g} z}\right) \omega^{*}+\frac{v}{D} \bar{\omega}^{*} .
\end{aligned}
$$

The boundary conditions follow from $(8 \mathrm{a})$ and $(8 \mathrm{~b})$ :

$$
v \omega^{*}(0)-D \bar{\omega}^{*}(0)=0 \text { and } v \omega^{*}\left(z_{m}\right)-D \bar{\omega}^{*}\left(z_{m}\right)=0 .
$$

Equations $(37 \& 38)$ are linear and homogeneous in $\omega^{*}$ and $\bar{\omega}^{*}$. Hence, if there exists a solution $\omega^{*}(z)$, then there also exists a solution $c \omega^{*}(z)$ for any arbitrary $c$. The free initial condition $\omega^{*}(0)$ is therefore arbitrary, and we may just as well work with $\omega^{*}(0)=1$. Therefore, using the first boundary condition in (38), the initial values for the two ODEs in (37) can be defined as

$$
\omega^{*}(0)=1 \quad \text { and } \quad \bar{\omega}^{*}(0)=\frac{v}{D} .
$$

Thus, we obtain the numerical recipe described below.

\subsection{Numerical recipe}

Starting from the initial conditions given in (39), the system of ODEs (37) can be integrated forward in $z$ until the solution hits upon either $v \omega^{*}(z)-D \bar{\omega}^{*}(z)=0$ or $\omega^{*}(z)=0$.

If we find $v \omega^{*}(z)-D \bar{\omega}^{*}(z)=0$ at some depth $z$, then the second boundary condition in (38) is satisfied. Hence, this depth $z$ is either the critical depth or the compensation depth. 
Alternatively, if we find $\omega^{*}(z)=0$ at some depth $z$, then the second boundary condition in (38) cannot be satisfied with positive $\omega^{*}(z)$. Hence, the critical depth and compensation depth do not exist. That is, we are somewhere in the parameter region between the minimal turbulence and maximal turbulence.

Repeating this procedure for various values of $D$, and ploting the critical depth or compensation depth whenever they exist, yields the graphs in Figure 4.

As a check, we compared the predictions of this numerical algorithm against the stationary results obtained by simulation of the full integro-PDE. Both methods always yielded the same critical depths and the same critical turbulences, but the numerical algorithm described in this section is orders of magnitude faster than simulation of the integro-PDE.

We emphasize that the approach outlined in this section is useful if one is solely interested in the values of the critical depths and critical turbulences. If one is also interested in the time evolution of the population density distributions, or in the particular shape of a stationary depth profile, then one should resort to the full simulation approach described in Section 6.

\section{Discussion}

It is not difficult to understand how sinking phytoplankton can maintain populations in optically shallow waters. In shallow waters, phytoplankton populations might sink to the bottom sediment, and light conditions near the bottom sediment may still be sufficient to sustain these populations. The question here is how sinking phytoplankton species can persist in deep waters, like the oceans, as well. The key finding in this paper is the existence of a 'turbulence window' that allows the persistence of sinking phytoplankton populations in deep waters (see Figure $4 \mathrm{~A}$ and Figure 5). If turbulence levels are less than a minimal turbulence, sinking rates dominate over growth rates and mixing rates. In this case, the phytoplankton population sinks downwards, and is lost from the euphotic zone. If turbulence levels exceed a maximal turbulence, vertical mixing rates dominate over growth and sinking. In this case, the phytoplankton population is uniformly mixed, and receives insufficient light in the deeper parts of the water column to persist. At intermediate turbulence levels, however, growth rates in the euphotic zone may exceed both sinking losses and mixing rates. Under these circumstances, a population of sinking phytoplankton may develop in the upper part of the water column. Thus, at intermediate turbulence levels, sinking phytoplankton species are capable to maintain a population within the euphotic zone.

We wish to emphasize that our analysis assumes light-limited growth of phytoplankton. This implies that the parameter space that permits bloom development in our model analysis indicates the maximum parameter space for blooms of sinking phytoplankton. In reality, conditions for bloom development will frequently be confined to a smaller subset within this parameter region, because of nutrient limitation, virus attack, or zooplankton grazing. However, conditions for bloom development can never exceed this maximum parameter space, because the available light energy is insufficient to sustain sinking phy- 
toplankton beyond these limits.

Our results indicate that arguments of previous authors (e.g., [14]) that there must be a strong selection pressure against sinking phytoplankton species need not hold. At least, these arguments need not hold for deep waters whose characteristics fall within the turbulence window, since deep waters located within the turbulence window can sustain sinking phytoplankton. The turbulence window will disappear, however, if the sinking velocity of phytoplankton is pushed beyond a certain threshold value (Fig. 4B). Thus, in line with intuitive reasoning, sinking phytoplankton cannot persist in deep waters if their sinking speed is too high; only phytoplankton with low to moderate sinking velocities can be sustained.

It is interesting to compare our findings with the earlier theoretical concepts developed by Riley et al. [23], Sverdrup [28], and Huisman et al. [11]. The present paper shows that these concepts are neither overlapping nor mutually exclusive. Instead, these earlier concepts form different elements in one coherent theory. The critical depth in our figures is equivalent to Sverdrup's critical depth $[28,21]$. The minimal turbulence in our figures corresponds to the classic relation, in Equation (19), derived by Riley et al. [23, page 90] (see also Shigesada and Okubo [25]). The maximal turbulence in our figures is equivalent to the critical turbulence found by Huisman et al. [11, 12]. Thus we have shown that the different concepts developed by these earlier authors can be integrated into a single unifying theory.

\section{References}

[1] K.R. Arrigo, D.H. Robinson, D.L. Worthen, R.B. Dunbar, G.R. DiTullio, M. VanWoert, and M.P. Lizotte, Phytoplankton community structure and the drawdown of nutrients and $\mathrm{CO}_{2}$ in the Southern Ocean, Science 283, 365-367 (1999).

[2] P.N. Brown, G.D. Byrne, and A.C. Hindmarsh, VODE: a variable-coefficient ODE solver, SIAM Journal on Scientific and Statistical Computing 10, 1038-1051 (1989).

[3] K.L. Denman and M.A. Pena, A coupled 1-D biological/physical model of the northeast subarctic Pacific Ocean with iron limitation, Deep-Sea Research II, 46, 2877-2908 (1999).

[4] G.R. DiTullio, J.M. Grebmeier, K.R. Arrigo, M.P. Lizotte, D.H. Robinson, A. Leventer, J.P. Barry, M.L. VanWoert, and R.B. Dunbar, Rapid and early export of Phaeocystis antarctica blooms in the Ross Sea, Antarctica, Nature 404, 595-598 (2000).

[5] P.L. Donaghay and T.R. Osborn, Toward a theory of biological-physical control of harmful algal bloom dynamics and impacts, Limnology and Oceanography 42, 12831296 (1997).

[6] U. Ebert, M. Arrayás, N.M. Temme, B.P. Sommeijer, and J. Huisman, Critical conditions for phytoplankton blooms, in preparation.

[7] P.G. Falkowski, R.T. Barber, and V. Smetacek, Biogeochemical controls and feedbacks on ocean primary production, Science 281, 200-206 (1998). 
[8] E. Hairer and G. Wanner, Solving Ordinary Differential Equations II, Stiff and Differential-algebraic Problems, 2nd ed., Springer Series in Computational Mathematics 14, Springer Verlag, Berlin (1996).

[9] C. Hirsch, Numerical Computation of Internal and External Flows I, Fundamentals of Numerical Discretization, Wiley and Sons, Chichester (1988).

[10] J. Huisman, Population dynamics of light-limited phytoplankton: microcosm experiments, Ecology 80, 202-210 (1999).

[11] J. Huisman, P. van Oostveen, and F.J. Weissing, Critical depth and critical turbulence: two different mechanisms for the development of phytoplankton blooms, Limnology and Oceanography 44, 1781-1788 (1999).

[12] J. Huisman, P. van Oostveen, and F.J. Weissing, Species dynamics in phytoplankton blooms: incomplete mixing and competition for light, American Naturalist 154, 46-68 (1999).

[13] J. Huisman and F.J. Weissing, Light-limited growth and competition for light in wellmixed aquatic environments: an elementary model, Ecology 75, 507-520 (1994).

[14] G.E. Hutchinson, A Treatise on Limnology II, Introduction to Lake Biology and the Limnoplankton, Wiley and Sons, New York (1967).

[15] B.M. Jamart, D.F. Winter, K. Banse, G.C. Anderson, and R.K. Lam, A theoretical study of phytoplankton growth and nutrient distribution in the Pacific Ocean off the Northwest U.S. coast, Deep-Sea Research 24, 753-773 (1977).

[16] J.T.O. Kirk, Light and Photosynthesis in Aquatic Ecosystems, 2nd ed., Cambridge Univ. Press, Cambridge (1994).

[17] L.D. Landau and E.M. Lifshitz, Course in Theoretical Physics, Vol. 5: Statistical Physics, 2nd ed., Pergamon Press, Oxford (1969).

[18] L.V. Lucas, J.E. Cloern, J.R. Koseff, S.G. Monismith, and J.K. Thompson, Does the Sverdrup critical depth model explain bloom dynamics in estuaries?, Journal of Marine Research 56, 375-415 (1998).

[19] K.H. Mann and J.R.N. Lazier, Dynamics of Marine Ecosystems: Biological-physical Interactions in the Oceans, 2nd ed., Blackwell, Oxford (1996).

[20] J. Monod, La technique de culture continue, théorie et applications, Annales de l'Institut Pasteur (Paris) 79, 390-410 (1950).

[21] T. Platt, D.F. Bird, and S. Sathyendranath, Critical depth and marine primary production, Proceedings of the Royal Society of London, B. 246, 205-217 (1991).

[22] C.S. Reynolds, The Ecology of Freshwater Phytoplankton, Cambridge University Press, Cambridge (1984). 
[23] G.A. Riley, H. Stommel, and D.F. Bumpus, Quantitative ecology of the plankton of the western North Atlantic, Bulletin of the Bingham Oceanographic Collection 12, 1-169 (1949).

[24] J. Sharples and P. Tett, Modelling the effect of physical variability on the midwater chlorophyll maximum, Journal of Marine Research 52, 219-238 (1994).

[25] N. Shigesada and A. Okubo, Analysis of the self-shading effect on algal vertical distribution in natural waters, Journal of Mathematical Biology 12, 311-326 (1981).

[26] D. Slagstad and K. Støle-Hansen, Dynamics of plankton growth in the Barents Sea: model studies, Polar Research 10, 173-186 (1991).

[27] T.J. Smayda, The suspension and sinking of phytoplankton in the sea, Oceanography and Marine Biology Annual Review 8, 353-414 (1970).

[28] H.U. Sverdrup, On conditions for the vernal blooming of phytoplankton, Journal du Conseil Permanent International pour l'Exploration de la Mer 18, 287-295 (1953). 